\title{
Nambu-Jona-Lasinio Version of Magnetic Monopole Physics with Dual Dirac Strings
}

\author{
M. Faber \\ A. N. Ivanov \\ W. Kainz \\ N.I. Troitskaya
}

Vienna, Preprint ESI 288 (1995)

November 29, 1995

Supported by Federal Ministry of Science and Research, Austria Available via http://www.esi.ac.at 


\title{
Nambu-Jona-Lasinio version of magnetic monopole physics with dual Dirac strings
}

\author{
M. Faber , A. N. Ivanov ${ }^{1}$, W. Kainz , N.I. Troitskaya ${ }^{1}$ \\ Institut für Kernphysik, Technische Universität Wien, \\ Wiedner Hauptstr. 8-10, A-1040 Vienna, Austria
}

\begin{abstract}
Magnetic monopoles are considered as massless fermion fields interacting via local four-monopole interaction of the Nambu-Jona-Lasinio kind leading to monopole condesation. Condensation of magnetic monopole currents and any derivative of them are obtained. It is shown that the bosonized version of this Monopole-NambuJona-Lasinio (MNJL) model is reducing to London's theory of dual superconductivity within Dirac's extension of Maxwell's Electrodynamics. The affinity of the MNJL-model with Compact Quantum Electrodynamics is discussed.
\end{abstract}

\footnotetext{
${ }^{1}$ Permanent Address: State Technical University, Department of Theoretical Physics, 195251 St. Petersburg, Russian Federation.
} 


\section{Introduction}

It is well-known that QCD possesses a confined phase being responsible for quark confinement. Most likely the phenomenon of quark confinement is closely connected with the properties of the nonperturbative vacuum of low-energy QCD. At present a qualitative description of quark confinement within QCD is far from being complete. Therefore, investigations of different models being much simpler than QCD and possessing confined phases should be rather important for the clarification of the mechanism of quark confinement at both qualitative and quantitative level.

Compact Quantum Electrodynamics (CQED) defined for lattices as nonlinear $U(1)$ gauge theory has a confined phase like QCD [1] and realizes confinement of electric charges. Thereby the investigation of CQED should aid understanding of quark confinement. As has been shown in [2] the nonperturbative vacuum of CQED behaves like an effective dual superconductor with magnetic monopoles. Due to magnetic monopoles the electric field flux between quarks rearranges and looks like a dual Dirac string. As a result quarks interact via a linearly rising potential $[3,4]$ that realizes quark confinement $[5,6]$ and spontaneous breaking of chiral symmetry $(\mathrm{SB} \chi \mathrm{S})[7]$ by a Nambu-Jona-Lasinio (NJL) type [8-14].

The NJL model should be considered as some kind of a relativistic extension of the BCS (Bardeen-Cooper-Schrieffer) theory of superconductivity [15]. Thereby the nonperturbative vacuum of the NJL model should possess the properties of the ground state of a superconductor in the superconducting phase. Thus if one would like to get a relativistic field theory with a nonperturbative vacuum that possesses the properties of dual superconductors one would put in the foundation of this theory the NJL model.

In this paper we conceive in continuum space-time a NJL version of magnetic monopole physics with dual Dirac strings, which we call Monopole Nambu-Jona-Lasinio (MNJL) model. It shows nonperturbative peculiarities of CQED in the confined phase. In particular it should possess a nonperturbative vacuum with properties of dual superconductors.

The Lagrangian of the starting system involving magnetic monopolos, quarks and dual-vector fields should read

$$
\begin{aligned}
\mathcal{L}(x)= & -\frac{1}{4} F_{\mu \nu}(x) F^{\mu \nu}(x)+ \\
& +\bar{\chi}(x) i \gamma^{\mu}\left(\partial_{\mu}+i g_{0} C_{\mu}(x)\right) \chi(x)+G[\bar{\chi}(x) \chi(x)]^{2}+ \\
& +\mathcal{L}_{\text {free quark }}(x) .
\end{aligned}
$$

where $\mathcal{L}_{\text {free quark }}(x)$ is a kinetic term for quark and antiquark

$$
\mathcal{L}_{\text {free quark }}(x)=-\sum_{i=q, \bar{q}} m_{i} \int d \tau\left(\frac{d X_{i}^{\mu}(\tau)}{d \tau} \frac{d X_{i}^{\nu}(\tau)}{d \tau} g_{\mu \nu}\right)^{1 / 2} \delta^{(4)}\left(x-X_{i}(\tau)\right) .
$$

We consider quark and antiquark as classical point-like particles with masses $m_{q}=m_{\bar{q}}=$ $m$, charges $Q_{q}=-Q_{\bar{q}}=Q_{0}$, and trajectories $X_{q}^{\nu}(\tau)$ and $X_{\bar{q}}^{\nu}(\tau)$, respectively. Note that $Q_{0}$ is a bare charge of a quark which will be renormalized by magnetic monopole interactions. The electric quark current $J^{\nu}(x)$ is given by

$$
J^{\nu}(x)=\sum_{i=q, \bar{q}} Q_{i} \int d \tau \frac{d X_{i}^{\nu}(\tau)}{d \tau} \delta^{(4)}\left(x-X_{i}(\tau)\right)
$$


Then $\chi(x)$ is a quantum fermionic monopole field, and $g_{0}$ is a bare magnetic charge connected with a bare electric charge of quark by the relation $g_{0} Q_{0}=2 \pi$. Following [16] we consider monopoles as fermions which are massless in the symmetry-unbroken phase. $G$ is a phenomenological coupling constant of a local four-monopole interaction. The field strength $F^{\mu \nu}(x)$ is defined [4]

$$
F^{\mu \nu}(x)=\mathcal{E}^{\mu \nu}(x)-{ }^{*}(d C(x))^{\mu \nu}
$$

where $(d C(x))^{\mu \nu}=\partial^{\mu} C^{\nu}(x)-\partial^{\nu} C^{\mu}(x)$, and ${ }^{*}(d C(x))^{\mu \nu}$ is a dual version

$$
{ }^{*}(d C(x))^{\mu \nu}=\frac{1}{2} \varepsilon^{\mu \nu \alpha \beta}(d C(x))_{\alpha \beta} \quad\left(\varepsilon^{0123}=1\right) .
$$

The electric strength field $\mathcal{E}^{\mu \nu}(x)$, induced by a dual Dirac string, is defined following $[3,17]$

$$
\mathcal{E}^{\mu \nu}(x)=Q \iint d^{2} v \delta^{(4)}(x-X) \sigma^{\mu \nu}(X)
$$

where we have denoted $d^{2} v=d \tau d \sigma$ and

$$
\sigma^{\mu \nu}(X)=\frac{\partial X^{\mu}}{\partial \tau} \frac{\partial X^{\nu}}{\partial \sigma}-\frac{\partial X^{\nu}}{\partial \tau} \frac{\partial X^{\mu}}{\partial \sigma} .
$$

Here $X^{\mu}=X^{\mu}(\tau, \sigma)$ represents the position of a point on the world sheet swept by the string. The sheet is parametrized by the internal coordinates $-\infty<\tau<\infty$ and $0 \leq \sigma \leq \pi$, so that $X^{\mu}(\tau, 0)=X_{-Q}^{\mu}(\tau)$ and $X^{\mu}(\tau, \pi)=X_{Q}^{\mu}(\tau)$ represent the world lines of an antiquark and a quark [3]. Within the definition (6) the tensor field $\mathcal{E}^{\mu \nu}(x)$ satisfies identically the equation of motion

$$
\partial_{\mu} F^{\mu \nu}(x)=J^{\nu}(x),
$$

that is the first pair of equations of motion of Dirac's extension of Maxwell's Electrodynamics. This means that the inclusion of a dual Dirac string in terms of $\mathcal{E}^{\mu \nu}(x)$ defined by (6) saturates completely the electric Gauss law.

The Lagrangian (1) is invariant under local $U(1)$ gauge transformations, where $U(1)$ is the Abelian gauge group of dual-vector potential transformations. Due to the fourmonopole interaction, proportional to the phenomenological positive coupling constant $G$, the local $U(1)$ symmetry gets broken spontaneously. In the symmetry-broken phase the $\chi$-field becomes massive and condensed. The process of the $\chi$-field condensation accompanies the appearance of a massive scalar $\bar{\chi} \chi$-collective excitation and the mass for the dual-vector field $C_{\mu}$. The longitudinal component of the massive dual-vector $C_{\mu}$-field is generated by the contribution of the massless Goldstone boson appearing due to the $U(1)$ symmetry breaking in accordance with the Goldstone theorem [18].

By varying the Lagrangian (1) with respect to $C_{\mu}(x)$ and $\bar{\chi}(x)$ we get the set of equations of motion

$$
\begin{gathered}
\partial_{\mu}{ }^{*} F^{\mu \nu}(x)=g_{0} \bar{\chi}(x) \gamma^{\nu} \chi(x) \\
\left(\partial_{\mu}+i g_{0} C_{\mu}(x)\right) \chi(x)=-2 G[\bar{\chi}(x) \chi(x)] \chi(x) .
\end{gathered}
$$


Eq.(8) can be valued as the second pair of equations of motion of Dirac's extension of Maxwell's Electrodynamics. The r.h.s. of eq.(8) should be identified with the magnetic current $j^{\nu}(x)$

$$
j^{\nu}(x)=g_{0} \bar{\chi}(x) \gamma^{\nu} \chi(x)
$$

The magnetic current $j^{\nu}(x)$ can be obtained in a standard way by applying local gauge transformation for the monopole field $\chi$

$$
\begin{aligned}
& \chi(x) \rightarrow \chi^{\prime}(x)=e^{i \alpha(x)} \chi(x), \\
& \bar{\chi}(x) \rightarrow \bar{\chi}^{\prime}(x)=\bar{\chi}(x) e^{-i \alpha(x)}
\end{aligned}
$$

and defining the magnetic current as a derivative of the Lagrangian (1) with respect to $\partial^{\mu} \alpha(x)$

$$
j_{\mu}(x)=-\frac{\delta \mathcal{L}(x)}{\delta \partial^{\mu} \alpha(x)} .
$$

For the subsequent investigation it is convenient to rewrite the Lagrangian (1) as follows

$$
\begin{aligned}
\mathcal{L}(x)= & -\frac{1}{4} \mathcal{E}_{\mu \nu}(x) \mathcal{E}^{\mu \nu}(x)+\frac{1}{2} \mathcal{E}_{\mu \nu}(x)^{*}(d C(x))^{\mu \nu}+ \\
& +\frac{1}{4}(d C(x))_{\mu \nu}(d C(x))^{\mu \nu}+ \\
& +\bar{\chi}(x) i \gamma^{\mu}\left(\partial_{\mu}+i g_{0} C_{\mu}(x)\right) \chi(x)+G[\bar{\chi}(x) \chi(x)]^{2}+ \\
& +\mathcal{L}_{\text {free quark }}(x) .
\end{aligned}
$$

Here we have used eq.(4). The Lagrangian (14) is the basic Lagrangian of the MNJL model which we apply to the description of magnetic monopole physics with dual Dirac strings.

The paper is organized as follows. In Sect. 1 we analyse the monopole condensation and derive in one-monopole loop approximation the effective Lagrangian of the $C_{\mu}$-field and the scalar $\sigma$-field being a collective excitation of the $\bar{\chi} \chi$ pair. In Sect. 2 we perform the complete bosonization of the $n$-point monopole Green function. In Sect. 3 we derive London equation of dual superconductivity. In Sect. 4 we represent the bosonized $n-$ point monopole Green function as a functional of a dual Dirac string shape. In Sect. 5 we evaluate the Green function of the massive dual-vector field. We show that the mechanism of the acquirement of the mass in one-monopole loop approximation is compared with that within the standard Higgs model. There is also no contradiction to the Goldstone theorem. In the Conclusion we discuss the obtained results.

\section{Monopole condensation}

Due to the strong attraction produced by the local four-monopole interaction the monopole field $\chi(x)$ acquires a mass $M$ satisfying the gap equation [8-14]

$$
M=-2 G<\bar{\chi}(0) \chi(0)>
$$


where

$$
<\bar{\chi}(0) \chi(0)>=-\frac{1}{4 \pi^{2}} M J_{1}(M)
$$

is the monopole condensate [8-14] and $J_{1}(M)$ is the quadratically divergent integral

$$
J_{1}(M)=\int \frac{d^{4} k}{\pi^{2} i} \frac{1}{M^{2}-k^{2}}=\Lambda^{2}-M^{2} \ln \left(1+\frac{\Lambda^{2}}{M^{2}}\right) .
$$

Here $\Lambda$ is the ultra-violet cut-off.

Monopole condensation accompanies the creation of a monopole-antimonopole $(\bar{\chi} \chi)$ collective excitation with the quantum numbers of a scalar Higgs meson field which we denote below as $\rho$-field.

Following [8-14] we determine the effective Lagrangian of the $\rho$-field and the massive $C_{\mu}$-field as follows

$$
\mathcal{L}_{\text {eff }}(x)=\tilde{\mathcal{L}}_{\text {eff }}(x)-\frac{\kappa^{2}}{4 G} \rho^{2}(x)
$$

where

$$
\hat{\mathcal{L}}_{\text {eff }}(x)=-i\left\langle x\left|\ln \frac{\operatorname{Det}(i \hat{\partial}-M+\Phi)}{\operatorname{Det}(i \hat{\partial}-M)}\right| x\right\rangle .
$$

Here we have denoted $\Phi=-g_{0} \gamma^{\mu} C_{\mu}-\kappa \sigma$, and $\sigma=\rho-M / \kappa$. The $\sigma$-field has vanishing vacuum expectation value (v.e.v.), i.e. $\langle\sigma\rangle=0$, while the v.e.v. of the $\rho$-field does not vanish, i.e. $\langle\rho\rangle=M / \kappa \neq 0$.

The effective Lagrangian $\tilde{\mathcal{L}}_{\text {eff }}(x)$ can be represented by an infinite series

$$
\tilde{\mathcal{L}}_{\text {eff }}(x)=\sum_{n=1}^{\infty} \frac{i}{n} \operatorname{tr}_{\mathrm{L}}\left\langle x\left|\left(\frac{1}{M-i \hat{\partial}} \Phi\right)^{n}\right| x\right\rangle=\sum_{n=1}^{\infty} \hat{\mathcal{L}}_{\mathrm{eff}}^{(n)}(x) .
$$

The index $\mathrm{L}$ means the evaluation of the trace over the Lorentz indices. The effective Lagrangian $\tilde{\mathcal{L}}_{\text {eff }}^{(n)}(x)$ is given by

$$
\begin{aligned}
\tilde{\mathcal{L}}_{\mathrm{eff}}^{(n)}(x)= & \int \prod_{\ell=1}^{n-1} \frac{d^{4} x_{\ell} d^{4} k_{\ell}}{(2 \pi)^{4}} \epsilon^{-i k_{1} \cdot x_{1}-\ldots-i k_{n} \cdot x}\left(-\frac{1}{n} \frac{1}{16 \pi^{2}}\right) \int \frac{d^{4} k}{\pi^{2} i} \times \\
& \times \operatorname{tr}_{\mathrm{L}}\left\{\frac{1}{M-\hat{k}} \Phi\left(x_{1}\right) \frac{1}{M-\hat{k}-\hat{k}_{1}} \Phi\left(x_{2}\right) \ldots\right. \\
& \left.\times \ldots \Phi\left(x_{n-1}\right) \frac{1}{M-\hat{k}-\hat{k}_{1}-\ldots-\hat{k}_{n-1}} \Phi(x)\right\}
\end{aligned}
$$

at $k_{1}+k_{2}+\ldots+k_{n}=0$. The r.h.s. of (27) describes the one-massive-monopole loop diagram with $n$-vertices. The monopole-loop diagrams with two vertices $(n=2)$ determine the kinetic term of the $\sigma$-field and give the contribution to the kinetic term of the $C_{\mu}$-field, while the diagrams with $(n \geq 3)$ describe the vertices of interactions of the $\sigma$ - and the $C_{\mu}$ - fields. In accordance with the prescription given in [9-14] the effective Lagrangian $\hat{\mathcal{L}}_{\text {eff }}(x)$ should be defined by the set of divergent one-massive-monopole-loop diagrams with $n=1,2,3$ and 4 vertices. The evaluation of these diagrams gives 


$$
\begin{aligned}
\mathcal{L}_{\mathrm{eff}}(x)= & \frac{1}{2} \frac{\kappa^{2}}{8 \pi^{2}} J_{2}(M) \partial_{\mu} \sigma(x) \partial^{\mu} \sigma(x)+M\left[\frac{\kappa}{2 G}-\frac{\kappa}{4 \pi^{2}} J_{2}(M)\right] \sigma(x)+ \\
& +\frac{1}{2}\left[-\frac{\kappa^{2}}{2 G}+\frac{\kappa^{2}}{4 \pi^{2}} J_{1}(M)-4 M \frac{\kappa^{2}}{8 \pi^{2}} J_{2}(M)\right] \sigma^{2}(x)- \\
& -2 M \kappa \frac{\kappa^{2}}{8 \pi^{2}} J_{2}(M) \sigma^{3}(x)-\frac{1}{2} \kappa^{2} \frac{\kappa^{2}}{8 \pi^{2}} J_{2}(M) \sigma^{4}(x)- \\
& -\frac{g_{0}^{2}}{48 \pi^{2}} J_{2}(M)(d C(x))_{\mu \nu}(d C(x))^{\mu \nu}- \\
& -\frac{g_{0}^{2}}{12 \pi^{2}}\left[J_{1}(M)+M^{2} J_{2}(M)\right] C_{\mu}(x) C^{\mu}(x) .
\end{aligned}
$$

In order to get a correct factor of the kinetic term of the $\sigma$-field we have to put [9-14]

$$
\frac{\kappa^{2}}{8 \pi^{2}} J_{2}(M)=1
$$

where $J_{2}(M)$ is a logarithmically divergent integral

$$
J_{2}(M)=\int \frac{d^{4} k}{\pi^{2} i} \frac{1}{M^{2}-k^{2}}=\ln \left(1+\frac{\Lambda^{2}}{M^{2}}\right)-\frac{\Lambda^{2}}{M^{2}+\Lambda^{2}} .
$$

Accounting to the gap equation (15) and the constraint (23) we bring the effective Lagrangian $(22)$ to the form

$$
\begin{aligned}
\mathcal{L}_{\text {eff }}(x)= & \frac{1}{2} \partial_{\mu} \sigma(x) \partial^{\mu} \sigma(x)-\frac{1}{2} M_{\sigma}^{2} \sigma^{2}(x)\left[1+\kappa \frac{\sigma(x)}{M_{\sigma}}\right]^{2}+ \\
& -\frac{g_{0}^{2}}{48 \pi^{2}} J_{2}(M)(d C(x))_{\mu \nu}(d C(x))^{\mu \nu}- \\
& -\frac{g_{0}^{2}}{12 \pi^{2}}\left[J_{1}(M)+M^{2} J_{2}(M)\right] C_{\mu}(x) C^{\mu}(x) .
\end{aligned}
$$

where $M_{\sigma}=2 M$ is the mass of the $\sigma$-field.

The term proportional to $(d C(x))_{\mu \nu}(d C(x))^{\mu \nu}$ can be removed by the renormalization of the wave-function of the $C_{\mu}$-field with the subsequent renormalization of the magnetic charge $g_{0}$. We leave to readers to get convinced in this assertion by performing these quantum field theory exercises and emphasize that below $g$ means the renormalized magnetic charge connected with $g_{0}$ by the expression

$$
g^{2}=g_{0}^{2}\left[1-\frac{g_{0}^{2}}{12 \pi^{2}} J_{2}(M)\right]^{-1}=g_{0}^{2}\left[1+\frac{2}{3} \frac{g_{0}^{2}}{\kappa^{2}}+\ldots\right] .
$$

Here we have applied the constraint (23). The expansion in the r.h.s. of (26) is valid if $\kappa \gg g$.

After the integration over the monopole degrees of freedom the total Lagrangian should read

$$
\mathcal{L}_{\text {tot }}(x)=-\frac{1}{4} \mathcal{E}_{\mu \nu}(x) \mathcal{E}^{\mu \nu}(x)+\frac{1}{2}{ }^{*} \mathcal{E}_{\mu \nu}(x)(d C(x))^{\mu \nu}-
$$




$$
\begin{aligned}
& +\frac{1}{4}(d C(x))_{\mu \nu}(d C(x))^{\mu \nu}-\frac{1}{2} M_{C}^{2} C_{\mu}(x) C^{\mu}(x)+ \\
& +\frac{1}{2} \partial_{\mu} \sigma(x) \partial^{\mu} \sigma(x)-\frac{1}{2} M_{\sigma}^{2} \sigma^{2}(x)\left[1+\kappa \frac{\sigma(x)}{M_{\sigma}}\right]^{2}+ \\
& +\mathcal{L}_{\text {free quark }}(x) .
\end{aligned}
$$

where

$$
M_{C}^{2}=\frac{g^{2}}{6 \pi^{2}}\left[J_{1}(M)+M^{2} J_{2}(M)\right]=\frac{g^{2}}{6 \pi^{2}} \frac{\Lambda^{4}}{M^{2}+\Lambda^{2}} .
$$

We identify $M_{C}$ with the mass of the $C_{\mu}$-field in the phase of the spontaneously broken $U(1)$-symmetry. The longitudinal component of the massive $C_{\mu}$-field is generated by the contribution of massless Goldstone bosons the appearance of which accompanies with the spontaneous breaking of the $U(1)$ gauge symmetry in accordance with the Goldstone theorem [18]. A more detailed derivation of the Green function of the massive $C_{\mu}$-field displaying the contribution of Goldstone bosons is given in Sect. 5 .

It should be emphasized that the field strength $\mathcal{E}^{\mu \nu}(x)$ in the Lagrangian (27) contains a renormalized electric charge of a quark $Q$, i.e. $Q=\left(g_{0} / g\right) Q_{0}$.

\section{Monopole Green functions}

The phenomenon of the magnetic current condensation has been observed within the framework of CQED by DeGrand and Toussaint [19]. For the analysis of this phenomenon within the MNJL model we have to consider the v.e.v. of time-ordered products of densities expressed in terms of the massless-monopole fields, i.e. the monopole Green function

$$
G\left(x_{1}, \ldots, x_{n}\right)=<0\left|\mathrm{~T}\left(\bar{\chi}\left(x_{1}\right) \Gamma_{1} \chi\left(x_{1}\right) \ldots \bar{\chi}\left(x_{n}\right) \Gamma_{n} \chi\left(x_{n}\right)\right)\right| 0>_{\text {connected }}
$$

where $\Gamma_{i}(i=1, \ldots, n)$ are the Dirac matrices.

Following the derivation suggested in [13] one can represent the r.h.s. of (29) in the following form

$$
\begin{aligned}
& G\left(x_{1}, \ldots, x_{n}\right)= \\
& =\quad<0\left|\mathrm{~T}\left(\bar{\chi}\left(x_{1}\right) \Gamma_{1} \chi\left(x_{1}\right) \ldots \bar{\chi}\left(x_{n}\right) \Gamma_{n} \chi\left(x_{n}\right)\right)\right| 0>_{\text {connected }}= \\
& ={ }^{(M)}<0 \mid \mathrm{T}\left(\bar{\chi}_{M}\left(x_{1}\right) \Gamma_{1} \chi_{M}\left(x_{1}\right) \ldots \bar{\chi}_{M}\left(x_{n}\right) \Gamma_{n} \chi_{M}\left(x_{n}\right) \times\right. \\
& \quad \times \exp i \int d^{4} x\left[\mathcal{L}_{\text {int }}\left(\chi_{M}(x), \sigma(x), C_{\mu}(x)\right)+\right. \\
& \left.\left.\quad+\mathcal{L}_{\text {tot }}\left(\sigma(x), C_{\mu}(x)\right)-\mathcal{L}^{(0)}\left(\sigma(x), C_{\mu}(x)\right)\right]\right) \mid 0>_{\text {connected }}^{(M)}
\end{aligned}
$$

where

$$
\mathcal{L}_{\text {int }}\left(\chi_{M}(x), \sigma(x), C_{\mu}(x)\right)=-g \bar{\chi}_{M}(x) \gamma^{\nu} \chi_{M}(x) C_{\nu}(x)-\kappa \bar{\chi}_{M}(x) \chi_{M}(x) \sigma(x)
$$

and $\mathcal{L}^{(0)}\left(\sigma(x), C_{\mu}(x)\right)$ is the Lagrangian of the free massive $\sigma-$ and $C_{\mu}-$ fields. Then $\mid 0>^{(M)}$ is the wave-function of the nonperturbative vacuum in the symmetry-broken 
phase. The complete coincidence of the N.J model with the BCS-theory of superconductivity admits the exact evaluation of $\mid 0>^{(M)}$ in the form $[8,15]$

$$
\left|0>^{(M)}=\prod_{\vec{p}, \lambda= \pm 1}\left[\sqrt{\frac{1+\beta_{\vec{p}}}{2}}+\lambda \sqrt{\frac{1-\beta_{\vec{p}}}{2}} a^{(0) \dagger}(\vec{p}, \lambda) b^{(0) \dagger}(-\vec{p}, \lambda)\right]\right| 0>^{(0)}
$$

where $\vec{\beta}_{\vec{p}}=\vec{p} / E_{\vec{p}}=\vec{p} / \sqrt{\vec{p}^{2}+M^{2}}$ is the velocity of massive monopoles with the mass $M$, and $a^{(0) \dagger}(\vec{p}, \lambda)$ (or $b^{(0) \dagger}(-\vec{p}, \lambda)$ ) denotes the creation operator of a massless monopole (or antimonopole) with the momentum $\vec{p}$ and helicity $\lambda ;\left|0>{ }^{(0)} \equiv\right| 0>$ is the wave-function of the vacuum in the $U(1)$-symmetry phase. In the linear approximation the operators of annihilation and creation of massive monopoles (or antimonopoles) are connected with the corresponding operators annihilating and creating massless monopoles (or antimonopoles) via the Bogoliubov transformation

$$
\begin{aligned}
a^{(M)}(\vec{p}, \lambda) & =a^{(0)}(\vec{p}, \lambda) \sqrt{\frac{1+\beta_{\vec{p}}}{2}}+b^{(0) \dagger}(-\vec{p}, \lambda) \lambda \sqrt{\frac{1-\beta_{\vec{p}}}{2}}, \\
b^{(M)}(-\vec{p}, \lambda) & =b^{(0)}(-\vec{p}, \lambda) \sqrt{\frac{1+\beta_{\vec{p}}}{2}}+a^{(0) \dagger}(\vec{p}, \lambda) \lambda \sqrt{\frac{1-\beta_{\vec{p}}}{2}} .
\end{aligned}
$$

In order to pick up the phenomenon of magnetic current condensation we have to consider the following v.e.v.

$$
<0\left|j_{\nu}(x) j^{\nu}(x)\right| 0>
$$

where the magnetic current $j^{\nu}(x)$ is defined by formula (11) with a renormalized magnetic charge $g$.

In terms of the massive-monopole fields interacting with the $\sigma$-field and the massive $C_{\mu}$-field the v.e.v. (34) is given by

$$
\begin{aligned}
<0\left|j_{\nu}(x) j^{\nu}(x)\right| 0>= & (M)<0 \mid \mathrm{T}\left(g^{2} \bar{\chi}_{M}(x) \gamma_{\nu} \chi_{M}(x) \bar{\chi}_{M}(x) \gamma^{\nu} \chi_{M}(x) \times\right. \\
& \times \exp i \int d^{4} x^{\prime}\left[\mathcal{L}_{\mathrm{int}}\left(\chi_{M}\left(x^{\prime}\right), \sigma\left(x^{\prime}\right), C_{\mu}\left(x^{\prime}\right)\right)+\right. \\
& \left.\left.+\mathcal{L}_{\text {tot }}\left(\sigma\left(x^{\prime}\right), C_{\mu}\left(x^{\prime}\right)\right)-\mathcal{L}^{(0)}\left(\sigma\left(x^{\prime}\right), C_{\mu}\left(x^{\prime}\right)\right)\right]\right) \mid 0>_{\text {connected }}^{(M)} .
\end{aligned}
$$

In the tree-meson approximation the r.h.s. of the v.e.v. (34) is defined by the monopole condensate

$$
<0\left|j_{\nu}(x) j^{\nu}(x)\right| 0>=-g^{2}[<\bar{\chi}(0) \chi(0)>]^{2}
$$

where the monopole condensate $\langle\bar{\chi}(0) \chi(0)\rangle$ is given by formula (15). Thus in the MNJL model the magnetic current condensation exists and is fully due to the magnetic monopole condensation.

As has been shown in [20] CQED predicts the nonzero v.e.v. of the curl of the magnetic current, i.e. the quantity

$$
<0\left|(d j(x))_{\mu \nu}(d j(x))^{\mu \nu}\right| 0>
$$


should have a nonvanishing value. The direct evaluation of (37) in the tree-meson approximation gives

$$
<0\left|(d j(x))_{\mu \nu}(d j(x))^{\mu \nu}\right| 0>=-\frac{g^{2}}{128 \pi^{4}}\left[\Lambda^{8}-16 \Lambda^{4} M^{2} J_{1}(M)+28 M^{4} J_{1}^{2}(M)\right] .
$$

The analogous calculation one can perform for the v.e.v. of any order derivatives of magnetic currents

$$
<0\left|\partial_{\mu_{1}} \ldots \partial_{\mu_{n}} j(x) \partial^{\mu_{1}} \ldots \partial^{\mu_{n}} j(x)\right| 0>
$$

This implies that within the MNJL model the v.e.v. (39) is fully defined in terms of the cut-off $\Lambda$ and the monopole mass $M$. The dependence of any v.e.v. on a dual Dirac string shape can be also taken into account (see Sect. 4).

\section{London's equation of dual superconductivity}

In order to show that the MNJL model admits the London equation of dual superconductivity we have to evaluate the v.e.v. of the magnetic current, i.e.

$$
\begin{aligned}
& \mathcal{J}^{\nu}(x)=<0\left|j_{\nu}(x)\right| 0>= \\
& ={ }^{(M)}<0 \mid \mathrm{T}\left(\overline { \chi } _ { M } ( x ) \gamma _ { \nu } \chi _ { M } ( x ) \operatorname { e x p } i \int d ^ { 4 } x ^ { \prime } \left[\mathcal{L}_{\text {int }}\left(\chi_{M}\left(x^{\prime}\right), \sigma\left(x^{\prime}\right), C_{\mu}\left(x^{\prime}\right)\right)+\right.\right. \\
& \left.\left.+\mathcal{L}_{\text {tot }}\left(\sigma\left(x^{\prime}\right), C_{\mu}\left(x^{\prime}\right)\right)-\mathcal{L}^{(0)}\left(\sigma\left(x^{\prime}\right), C_{\mu}\left(x^{\prime}\right)\right)\right]\right) \mid 0>_{\text {connected }}^{(M)} .
\end{aligned}
$$

In the tree-meson approximation the r.h.s. of (40) can be expessed in terms of the $C_{\mu^{-}}$ field

$$
\mathcal{J}^{\nu}(x)=-M_{C}^{2} C^{\nu}(x)
$$

This is a relativistic extension of the London's equation of dual superconductivity [3,4].

The relation (40) can be obtained directly from the Lagrangian (27) by performing an infinitesimal gauge transformation of the $C_{\mu}$-field

$$
C_{\mu}(x) \rightarrow C_{\mu}^{\prime}(x)=C_{\mu}(x)-\partial_{\mu} \alpha(x)
$$

and defining the magnetic current as a derivative of the Lagrangian (27) over $\partial_{\mu} \alpha(x)$

$$
\mathcal{J}_{\mu}(x)=-\frac{\delta \mathcal{L}(x)}{\delta \partial^{\mu} \alpha(x)} .
$$

The r.h.s. of (43) is proportional to the $C^{\nu}$-field as it is given by eq.(41).

\section{Monopole Green functions as functionals of the dual string shape}

By varying the Lagrangian $\mathcal{L}_{\text {tot }}(x)$ with respect to $C_{\nu}(x)$ one obtains the equations of motion

$$
\left(\square+M_{C}^{2}\right) C^{\nu}(x)=-\partial_{\mu}{ }^{*} \mathcal{E}^{\mu \nu}(x)
$$


Here we have used the constraint $\partial_{\mu} C^{\mu}(x)=0$. The solution of eq.(44) reads [4]

$$
C^{\nu}[\mathcal{E}(x)]=-\int d^{4} x^{\prime} \Delta\left(x-x^{\prime}\right) \partial_{\mu}^{\prime *} \mathcal{E}^{\mu \nu}\left(x^{\prime}\right)
$$

where $\Delta\left(x-x^{\prime}\right)$ is the Green function of the $C_{\mu}$-field. The notation $C^{\nu}[\mathcal{E}(x)]$ means that the $C_{\mu}$-field is a functional of the dual string shape.

Substituting (45) in (30) we obtain the monopole Green function as a functional of $\mathcal{E}^{\mu \nu}(x)$, i.e. a dual Dirac string shape

$$
\begin{aligned}
& G\left(x_{1}, \ldots, x_{n} ; \mathcal{E}\right)= \\
& =<0\left|\mathrm{~T}\left(\bar{\chi}\left(x_{1}\right) \Gamma_{1} \chi\left(x_{1}\right) \ldots \bar{\chi}\left(x_{n}\right) \Gamma_{n} \chi\left(x_{n}\right)\right)\right| 0>_{\text {connected }}= \\
& ={ }^{(M)}<0 \mid \mathrm{T}\left(\bar{\chi}_{M}\left(x_{1}\right) \Gamma_{1} \chi_{M}\left(x_{1}\right) \ldots \bar{\chi}_{M}\left(x_{n}\right) \Gamma_{n} \chi_{M}\left(x_{n}\right) \times\right. \\
& \times \exp i \int d^{4} x\left\{-g \bar{\chi}_{M}(x) \gamma^{\nu} \chi_{M}(x) C_{\nu}[\mathcal{E}(x)]+\right. \\
& \left.\left.-\kappa \bar{\chi}_{M}(x) \chi_{M}(x) \sigma(x)-M_{\sigma} \kappa \sigma^{3}(x)-\frac{1}{2} \kappa^{2} \sigma^{4}(x)\right\}\right) \mid 0>_{\text {connected }}^{(M)} .
\end{aligned}
$$

The averaging over the string shape fluctuations should be performed with the weight $\exp i \mathcal{S}_{\text {string }}[\mathcal{E}]$, i.e.

$$
\mathcal{G}\left(x_{1}, \ldots, x_{n}\right)=\int \mathcal{D} \mu[\ell] e^{i \mathcal{S}_{\text {string }}[\mathcal{E}]} G\left(x_{1}, \ldots, x_{n} ; \mathcal{E}\right) .
$$

Here $\mathcal{D} \mu[\ell]$ is the measure of the integration over the shape of the dual Dirac string with the length $\ell[21]$, and $\mathcal{S}_{\text {string }}[\mathcal{E}]$ is the string action $[3,4]$

$$
\mathcal{S}_{\text {string }}[\mathcal{E}]=\frac{1}{4} M_{C}^{2} \int d^{4} x d^{4} x^{\prime} \mathcal{E}_{\mu \alpha}(x) \mathrm{D}_{\beta}^{\alpha}\left(x-x^{\prime}\right) \mathcal{E}^{\mu \beta}\left(x^{\prime}\right)
$$

where

$$
\mathrm{D}_{\beta}^{\alpha}\left(x-x^{\prime}\right)=\left(g_{\beta}^{\alpha}-2 \frac{\partial^{\alpha} \partial_{\beta}}{M_{C}^{2}}\right) \Delta\left(x-x^{\prime}\right) .
$$

Formula (47) is valid for the evaluation of any monopole Green function averaged over dual Dirac string-shape fluctuations.

\section{Green function of massive $C_{\mu}-$ field and Goldstone boson}

In this Section we give a derivation of the two-point Green function for the massive $C_{\mu}$-field and display the contribution of Goldstone bosons which accompany spontaneous breaking of $U(1)$ gauge symmetry as has been explained by the Goldstone theorem [18]. For simplicity we carry out the derivation in Lorentz gauge: $\partial_{\mu} C^{\mu}(x)=0$.

In accordance with quantum field theory [22] the two-point Green function of the massive $C_{\mu}$-field, calculated in the one-monopole-loop approximation, should be defined 
by the infinite series

$$
\begin{aligned}
i g^{2} D_{\mu \nu}(q) & =g_{0}^{2} \frac{1}{q^{2}+i 0}\left(g_{\mu \nu}-\frac{q_{\mu} q_{\nu}}{q^{2}}\right)+ \\
& +g_{0}^{2} \frac{1}{q^{2}+i 0}\left(g_{\mu \nu}-\frac{q_{\mu} q_{\alpha}}{q^{2}}\right) \Pi^{\alpha \beta}(q) \frac{1}{q^{2}+i 0}\left(g_{\beta \nu}-\frac{q_{\beta} q_{\nu}}{q^{2}}\right)+\ldots
\end{aligned}
$$

Here $\prod^{\alpha \beta}(q)$, being a tensor of vacuum polarization caused by the constribution of virtual magnetic monopole-antimonopole pairs, reads

$$
\begin{aligned}
& \Pi^{\alpha \beta}(q)=\frac{g_{0}^{2}}{16 \pi^{2}} \int \frac{d^{4} k}{\pi^{2} i} \operatorname{tr}\left\{\frac{1}{M-\hat{k}} \gamma^{\alpha} \frac{1}{M-\hat{k}-\hat{q}} \gamma^{\beta}\right\}= \\
& =\mathcal{A}\left(q^{2}\right)\left(q^{2} g^{\alpha \beta}-q^{\alpha} q^{\beta}\right)+\mathcal{B}\left(q^{2}\right) q^{\alpha} q^{\beta}= \\
& =\frac{g_{0}^{2}}{6 \pi^{2}}\left\{\left[J_{1}(M)+M^{2} J_{2}(M)\right]+\frac{1}{2} J_{2}(M) q^{2}\right\}\left(g^{\alpha \beta}-\frac{q^{\alpha} q^{\beta}}{q^{2}}\right)
\end{aligned}
$$

where

$$
\begin{aligned}
& \mathcal{A}\left(q^{2}\right)=\frac{g_{0}^{2}}{96 \pi^{2}}\left[-\frac{2}{q^{2}} J_{1}(M)+\frac{1}{q^{2}}\left(4 M^{2}-q^{2}\right) J_{2}(M)\right], \\
& \mathcal{B}\left(q^{2}\right)=\frac{g_{0}^{2}}{32 \pi^{2}}\left[-\frac{2}{q^{2}} J_{1}(M)+J_{2}(M)\right] .
\end{aligned}
$$

Eq.(51) represents the gauge invariant evaluation of the polarization tensor performed for the cut-off regularization.

Substituting (51) in (50) and summing up the infinite series one obtains

$$
\begin{aligned}
& i g^{2} D_{\mu \nu}(q)=g_{0}^{2}\left(g_{\mu \nu}-\frac{q_{\mu} q_{\nu}}{q^{2}}\right) \times \\
& \times\left[q^{2}-\frac{g_{0}^{2}}{6 \pi^{2}}\left\{\left[J_{1}(M)+M^{2} J_{2}(M)\right]+\frac{1}{2} J_{2}(M) q^{2}\right\}+i 0\right]^{-1}= \\
& =g_{0}^{2}\left(g_{\mu \nu}-\frac{q_{\mu} q_{\nu}}{q^{2}}\right) \times \\
& \times\left[q^{2}\left(1-\frac{g_{0}^{2}}{12 \pi^{2}} J_{2}(M)\right)-\frac{g_{0}^{2}}{6 \pi^{2}}\left[J_{1}(M)+M^{2} J_{2}(M)\right]+i 0\right]^{-1}= \\
& =\frac{g^{2}}{q^{2}-M_{C}^{2}+i 0}\left(g_{\mu \nu}-\frac{q_{\mu} q_{\nu}}{q^{2}}\right)= \\
& =\frac{g^{2}}{q^{2}-M_{C}^{2}+i 0}\left(g_{\mu \nu}-\frac{q_{\mu} q_{\nu}}{M_{C}^{2}}\right)+\frac{g^{2}}{M_{C}^{2}} \frac{q_{\mu} q_{\nu}}{q^{2}} .
\end{aligned}
$$

Here $g$ and $M_{C}$ are the renormalized magnetic charge of monopole and the mass of the $C_{\mu}$-field in the symmetry-broken phase given by eqs.(26) and (28), respectively. The term

$$
\frac{g^{2}}{M_{C}^{2}} \frac{q_{\mu} q_{\nu}}{q^{2}}
$$


in the r.h.s. of (53) describes the contribution of the massless Goldstone boson appearing due to spontaneous breaking of $U(1)$ gauge symmetry in accordance with the Goldstone theorem [18].

The evaluation of the two-point Green function of the massive $C_{\mu}$-field, given in the MNJL model, one should compare with that within the standard Higgs model [22] when a vector field acquires a mass in the tree approximation. In the case of the standard Higgs model $\Pi^{\alpha \beta}(q)$, defined in the tree approximation, is given by: $\Pi^{\alpha \beta}(q)=2 g_{0}^{2} v^{2} g^{\alpha \beta}$, where $v$ is the v.e.v. of the Higgs field. The infinite series (51) should be summed up to the form (53) with the vector meson mass equals $M_{\mathrm{V}}^{2}=2 g_{0}^{2} v^{2}$.

Thus the mechanism of the acquirement of mass by vector mesons, caused by spontaneous breaking of symmetry, given in the MNJL model and the standard Higgs model can be compared well. There is only the distinction that for the Higgs mechanism $g_{0}$ remains

unrenormalized. This implies that the quantity $M_{C}$ defined by eq.(28) is in reality the mass of the $C_{\mu}$-field in the symmetry-broken phase.

\section{Conclusion}

Now let us resume the obtained results. We have found that the NJL model applied to the description of monopole physics, i.e. the MNJL model, has turned out very useful for the understanding of the mechanism of monopole and magnetic current condensation. The local four-monopole interaction provides spontaneous breaking of the $U(1)$ gauge symmetry that leads to the monopole condensation and the appearance of massive monopole $\chi_{M}$-fields. They interact with the massive scalar monopole-antimonopole collective excitation $\sigma$-field and the massive dual-vector field $C_{\mu}$. As a result the v.e.v. of any time-ordered product of massless monopole densities can be evaluated in terms of v.e.v. of massive monopole densities with massive monopole fields interacting with the massive $\sigma$ - and the massive $C_{\mu}$-fields. This allows to reproduce in continuum space-time the condensation of any order derivative of the magnetic current, i.e.

$$
<0\left|\partial_{\mu_{1}} \ldots \partial_{\mu_{n}} j(x) \partial^{\mu_{1}} \ldots \partial^{\mu_{n}} j(x)\right| 0>
$$

observed within CQED. The v.e.v. can be evaluated in terms of dual Dirac string-shapes and averaged over string-shape fluctuations.

Thus, the enumerated peculiarities of the MNJL model, i.e. magnetic monopole condensation, the condensation of magnetic current and any order derivative of magnetic current and so on, should testify a close affinity between the MNJL model and CQED. This allows to think that the MNJL model can be considered as some kind of continuum space-time analogy of CQED.

The Nambu-Jona-Lasinio version of the monopole physics, i.e the MNJL-model, seems much more flexible in comparison with the dual Higgs model that we have suggested in ref.[23]. Indeed, in the MNJL-model the condensation of the magnetic current and its derivatives can be obtained in the main one-monopole-loop and in the tree $C_{\mu-}^{-}$ field approximation. In turn in the Dual Higgs model the condensation of these quantitites is the matter of the $C_{\mu}$-field loop contributions and does not appear in the tree $C_{\mu}$-field approximation. The more detail comparison of the MNJL-model with the dual Higgs model [23] we are planning to carry out in our forthcoming publications. 
With pleasure we acknowledge fruitful discussions with Prof. G. E. Rutkovsky. 


\section{References}

[1] P. Becher and H. Joos, Z. Phys. C15 (1982) 343.

[2] V. Singh, D. Browne and R. Haymaker, Phys. Rev. D47 (1993) 1715.

[3] Y. Nambu, Phys. Rev. D10 (1974) 4246.

[4] M. Faber, W. Kainz, A. N. Ivanov and N. I. Troitskaya, Phys. Lett. B344 (1995) 143 and references therein.

[5] A. N. Ivanov, N. I. Troitskaya, M. Faber, M. Schaler and M. Nagy, Phys. Lett. B336 (1994) 555 .

[6] A. N. Ivanov, N. I. Troitskaya and M. Faber, Nuovo. Cim. A108 (1995) 613.

[7] A. N. Ivanov, N. I. Troitskaya, M. Faber, M. Schaler and M. Nagy, Nuovo Cim. A107 (1994) 1667.

[8] Y. Nambu and G. Jona - Lasinio, Phys. Rev. 122 (1961) 345; ibid. 124 (1961) 246.

[9] T. Eguchi, Phys. Rev. D14 (1976) 2755;

K. Kikkawa, Progr. Theor. Phys. 56 (1976) 947;

H.Kleinert, Proc. of Int. Summer School of Subnuclear Physics, Erice 1976, Ed. A.Zichichi, p.289.

[10] T. Hatsuda and T. Kumihiro, Proc. Theor. Phys. 74 (1985) 765; Phys. Lett. B198 (1987) 126 ;

T. Kumihiro and T. Hatsuda, Phys. Lett. B206 (1988) 385.

[11] S. Klint, M. Lutz, V. Vogl and W. Weise, Nucl. Phys. A516 (1990) 429; 469 and references therein.

[12] A. N. Ivanov, M. Nagy and N. I. Troitskaya, Int. J. Mod. Phys. A7 (1992) 7305;

[13] A. N. Ivanov, Int. J. Mod. Phys. A8 (1993) 853.

[14] A. N. Ivanov, N. I. Troitskaya and M. Nagy, Int. J. Mod. Phys. A8 (1993) 2027.

A. N. Ivanov, N. I. Troitskaya and M. Nagy, Int. J. Mod. Phys. A8 (1993) 3425.

A. N. Ivanov, N. I. Troitskaya and M. Nagy, Phys. Lett. B308 (1993) 111;

A. N. Ivanov, N. I. Troitskaya and M. Nagy, Phys. Lett. B295 (1992) 308 ,

A. N. Ivanov and N. I. Troitskaya, Nuovo Cim. A108 (1995) 555.

[15] J. Bardeen, L. N. Cooper and J. R. Schrieffer: Phys. Rev. 106 (1957) 162; ibid. 108 (1957) 1175. 
[16] M. Blagoević and P. Senjanović, Phys. Rep. 157 (1988) 233 and references therein.

[17] P. A. M. Dirac, Proc. Roy. Soc. A133 (1931) 60; Phys. Rev. 74 (1948) 817.

[18] J. Goldstone, Nuovo Cim. 19 (1961) 154.

[19] T. A. DeGrand and D. Toussaint, Phys. Rev. D22 (1980) 2478.

[20] M. Zach, M. Faber, W. Kainz and P. Skala, Phys. Lett. B358 (1995) 325.

[21] M. Lüscher, K. Symanzik and P. Weisz, Nucl. Phys. B173 (1980) 365.

[22] C. Itzykson and J. - B. Zuber : Quantum Field Theory (McDraw - Hill) ,1980.

[23] M. Faber, A. N. Ivanov, W. Kainz and N. I. Troitskaya, Dual Higgs model with dual Dirac strings, ESI-Preprint, November 1995 (submitted to Nucl. Phys. B). 